\title{
Abducens nerve palsy following spinal anaesthesia
}

\author{
Panteli E., Sioulas N., Kolliopoulou G., Aretha D., Fili K., Fligou F.
}

Patras University Hospital, Dept of Anaesthesiology \& Intensive Care, Patras, Greece

Background: Cranial nerve $(\mathrm{CN})$ palsy is a rare complication of spinal anesthesia ( $1: 300$ to 1:8000). The abducens nerve (CN 6) is most frequently affected because of its long intracranial course. CN 6 palsy usually occurs 4 to 14 days post puncture, can be unilateral or bilateral and is usually associated with post lumbar puncture headache (PLPH).

Case report: A 67 years old ASA II woman underwent revision of total hip arthroplasty due to dislocation 3 days after the initial operation. The preoperative physical examination and laboratory testing was normal. A single shot spinal anesthesia was performed at the L2-L3 interspace with a 25G spinal needle and the injection of $12 \mathrm{mg}$ chirocaine $0.5 \%$ and $10 \gamma$ fentanyl. Surgery was uneventfull, but six hours later the patient complained for diplopia, without other neurological symptoms (headache, backache, sensory or motor deficits). Clinical examination revealed a right CN6 paresis, with binocular horizontal diplopia which was worse on right gaze. Blood laboratory tests, brain CT scan and MRI did not reveal any pathology. The review of the previous anesthetic chart showed that the patient had received spinal anaesthesia 3 days ago, at the same interspace with a $23 \mathrm{G}$ needle. The patient underwent weekly ophthalmological examination until diplopia resolved spontaneously after 32 days.

Discussion: CN6 palsy is a rare complication of spinal anaesthesia usually associated with PLPH that occurs 4 to 14 days after the block. In this case report, the patient did not develop PLPH, and the paresis occurred within 3 days and 6 hours after the first and second spinal shot respectively. It is suggested that the cerebrospinal fluid (CSF) leakage leads to intracranial hypotension, traction of the CN6 and subsequent nerve dysfunction. Therefore, it is possible that in this case, the CSF leakage after the second spinal was superimposed to the ongoing leakage caused by the first anaesthetic, although PLPH was not observed. A high index of suspicion is needed to diagnose potential rare complications in the postoperative peri.od. 